\title{
Regards
}

\section{L'environnement entre science et sens commun}

\author{
Marc Mormont \\ Sociologue, Université de Liège, Unité SEED, 6700 Arlon, Belgique
}

Après une longue éclipse d'un demi-siècle, le pragmatisme de John Dewey connaît un regain d'intérêt et de nouvelles traductions. Dewey est sans doute le philosophe de la connaissance qui prépare le mieux à réfléchir au statut de la connaissance dans les sociétés industrielles : c'est qu'il considère en même temps que la méthode scientifique a constitué une nouveauté radicale et irréversible dans l'histoire humaine et qu'elle n'a pour autant ni supériorité ni autorité de droit sur les autres rapports au réel. Refusant le dualisme entre nature et société, ou encore celui entre économie et morale, il anticipe un espace conceptuel pour la question de l'environnement, en même temps d'ailleurs qu'une proposition d'espace pour une interdisciplinarité qui devrait s'ancrer dans les « affaires » qui constituent la trame de la vie humaine. Cette note invite à relire Dewey comme un philosophe de l'environnement avant l'environnement.

Les questions environnementales - des rapports entre sciences et sociétés - interrogent la connaissance scientifique - son statut, sa portée, ses effets. John Dewey est surtout connu en France ${ }^{1}$ pour sa contribution au débat sur la démocratie. Mais comme de récentes traductions ${ }^{2} l^{\prime}$ indiquent, il était avant tout un philosophe de la connaissance dont il faisait un mode particulier de

\footnotetext{
1 Notons que Dewey fut bien introduit en France au début du siècle dernier puisque Durkheim lui consacra un cours entier à la Sorbonne en 1913-1914. Ce cours est disponible sur http:/ / classiques.uqac.ca/classiques/Durkheim_emile/ pragmatisme_et_socio/pragmatisme_et_socio.html.

2 En particulier, Expérience et nature, traduction de Joëlle Zask, Gallimard, 2012.
}

l'expérience ${ }^{3}$. Lionel Charles ${ }^{4}$ a déjà attiré l'attention sur la spécificité de l'approche des pragmatistes, et, en particulier, de la problématisation de Dewey des rapports à la nature. Un de ses derniers articles - dont nous traduisons ci-dessous quelques passages ${ }^{5}$ - fait ainsi explicitement référence à l'environnement dans des termes qui surprennent pour l'époque (1948).

«Ce qu'on appelle environnement, c'est là où les conditions dites physiques sont enchevêtrées aux conditions culturelles et sont, dès lors, plus que "physiques" au sens technique du terme. L'"environnement" n'est pas quelque chose qui entoure les activités humaines ou qui les concerne de l'extérieur. C'est leur medium, ou milieu au sens où un medium est un intermédiaire dans l'exécution ou la réalisation des activités humaines, aussi bien le canal dans lequel elles se déplacent que le véhicule qu'elles empruntent. Le rétrécissement de ce milieu est la cause directe de tout un appauvrissement inutile de la vie humaine; le seul sens dans lequel le terme de "social" mérite le respect est le sens qui fait de ce milieu, dans lequel la vie humaine se déroule, le milieu d'une vie plus riche. » (p. 185)

Cet article est paru dans le Journal of Philosophy sous le titre "Common sense and science: their respective

\footnotetext{
3 L'expérience est le concept central de la philosophie de Dewey au sens où la réalité est expérience. Voir Dewey, J., 2005. La réalité comme expérience, Tracés. Revue de Sciences humaines, http://traces.revues.org/204 (traduit de Dewey, J., 1906. Reality as experience, The Journal of Philosophy, Psychology and Scientific Methods, III, 10, 253-257).

4 Charles, L., 2007. Pragmatisme et environnement, in Lolive, J., Soubeyran, O. (Eds), L'émergence des cosmopolitiques, Paris, La Découverte, 272-283.

5 Traductions de l'auteur Marc Mormont. Les termes en italique ou entre guillemets sont de Dewey.
}

Auteur correspondant : mmormont@ulg.ac.be 
frames of reference ${ }^{6} »$. Dewey y pose la question des rapports entre sens commun et science. Il tente d'abolir la différence de nature qu'on y voit et même de rétablir l'indispensable recours au sens commun.

\section{Trans-action}

En mettant en avant cette conception de l'environnement comme « milieu », Dewey cherche en fait à annuler le dualisme - parmi d'autres, on y reviendra - qui sépare l'humanité du monde biophysique. L'idée de milieu, de medium, où interfèrent facteurs humains et facteurs naturels - qu'il mentionne comme non humains - peut paraître assez commune aujourd'hui, mais pour Dewey $c^{\prime}$ est avant tout une manière de dire non seulement qu'il est impossible de séparer ces facteurs enchevêtrés, mais surtout que, même si nous les distinguons - et il juge utile de le faire dans certaines conditions - l'important c'est de voir ce milieu comme fait de flux, d'actions réciproques, de devenirs s'impliquant mutuellement et qui constituent - au sens de donner constitution -, les composantes de ces milieux. Ici intervient un concept qui apparaît assez tôt dans les travaux de Dewey mais auquel il accorde une place centrale et cruciale dans ses derniers écrits ${ }^{7}$. Le concept de trans-action, il le dit explicitement, doit permettre de dépasser celui d'interaction. Que reproche-t-il à cette notion ? Selon lui, elle a été un grand progrès, elle a permis d'aller au-delà des conceptions «self-actional » qui attribuent à des entités des propriétés intangibles, immuables. La notion d'interaction a permis de mettre en évidence que les propriétés des objets dérivaient de leurs relations à d'autres objets. Mais, selon lui, ce concept maintient l'idée d'entités préalables à l'interaction.

«On peut ne remarquer l'air qui intervient dans la transaction respiratoire qu'en cas d'obstruction; il en va de même de la multitude des facteurs humains et non humains qui participent à ce que nous faisons, disons et pensons, même dans nos soliloques et nos rêves. » (p. 185)

Qu'est-ce qu'un poumon sans air, sans globules, sans oxygène, sans pompe cardiaque ? Il est un milieu, et ses «propriétés » ne sont que le résultat de conditions et de

6 Dewey, J., 1948. Common sense and science: their respective frames of reference, Journal of Philosophy 45, 8, 197-208. Repris dans le recueil Dewey, J., Bentley, A., 1949. Knowing and the known, Boston, Beacon Press, https: / /www.aier.org/sites/ default/files/Files/Documents/Standard/KnowingKnown Fullext.pdf. La pagination des passages traduits se réfère à cette édition.

7 Il développe principalement ce concept dans le recueil d'articles Dewey, J., Bentley, A.F., op. cit. conséquences qui permettent les flux qui constituent ce milieu. Ce concept de transaction n'a été que faiblement ou partiellement intégré dans les travaux de sciences de l'homme $^{8}$.

« [...] c'est que la vie humaine elle-même, à la fois individuellement et collectivement, est faite de transactions dans lesquelles les humains participent, avec des non-humains et d'autres humains, d'un milieu, si bien que, sans l'ensemble de ces humains et de ces non-humains, nous ne pourrions rester en vie ni même faire quoi que ce soit. » (p. 185)

La perspective transactionnelle ne nie évidemment pas que le sang n'est pas l'oxygène, mais elle affirme que le réel auquel nous avons affaire est fait des situations d'échange et de devenirs entre ces «éléments » qui n'acquièrent d'identités que dans le flux spécifique de la respiration. Adopter une perspective transactionnelle consiste donc à privilégier des situations, et c'est donc une méthode, une manière d'observer. Dewey insiste moins sur le caractère hétérogène (hybride, dirait-on aujourd'hui) de ces situations que sur le fait qu'elles intègrent dans des flux d'échange. Et le sens commun est constitué par ces situations qui se présentent comme des " affaires », des préoccupations, des concernements.

L'originalité de Dewey est de considérer aussi la connaissance - en tant que processus, knowing - comme une transaction, donc d'abord comme une action qui a des conditions et des conséquences. Il en fait un processus naturel autant que tous ceux à l'œuvre dans la nature ou dans la société. Il abolit le dualisme de l'observateur et de l'observé. La connaissance, telle que nous l'entendons souvent, communément, c'est-à-dire comme des énoncés qui résultent du processus de connaître, n'est qu'un aspect, $\mathrm{qu}^{\prime}$ une phase du cheminement de la connaissance, au même titre que le poumon n'est qu'une phase du processus de respiration. Par conséquent, ce qui est au centre du connaître, ce sont les médiations que l'observateur déploie pour connaître, médiations qui vont produire, dans un même mouvement, le connaissant et le connu. Ces médiations, ces opérations ont aussi des conditions, et, parmi elles, les connaissances antérieures, les instruments techniques ou intellectuels; la connaissance a aussi des conséquences qui d'ailleurs intéressent l'observateur, et qui vont évidemment faire retour sur les connaissances antérieures, pour les confirmer, les infirmer ou les reformuler. Dans cette perspective, il n'y a pas donc pas, d'un côté, un esprit, une conscience, une rationalité et, de l'autre, une réalité.

\footnotetext{
8 On le retrouve bien dans les travaux de l'économiste institutionnaliste Commons ou dans la sociologie de la transaction sociale (voir le numéro de Pensée plurielle « Penser et agir dans l'incertain : l'actualité de la transaction sociale », $\mathrm{n}^{\circ} 33-34$, 2013/2-3).
} 


\section{Sens commun et science}

De ce point de vue, sens commun (qu'on pourrait aussi appeler culture au sens socioanthropologique) et science sont tous deux des processus transactionnels. Dans le sens commun, ancré dans le cours de la vie des communautés humaines, les situations, les problèmes suscitent aussi bien le désir de connaître que le désir d'agir. Dewey met sur le même plan les démarches du sens commun et de la science dans le rapport au monde. Pour les deux démarches, se présentent des questions inscrites dans le cours des choses, des « affaires », et ces questions sont des concernements.

"Tous ces termes "affaire, soin, attention, chose", etc. fusionnent dans une unité de différents sens qui peuvent être distingués comme sens émotionnel ou sens intellectuel ou sens pratique, les deux premiers étant par ailleurs des traits toujours présents dans le troisième. Sauf en contexte, il n'est d'ailleurs pas possible de dire lequel est le plus prégnant. Et, en contexte, c'est toujours une question de degré, jamais de séparation. » (p. 187)

Dès lors, pour Dewey, la recherche est une activité comme les autres, mobilisant toutes les dimensions de toute activité, émotionnelle, intellectuelle et pratique. Croire qu'on pourrait assigner émotion au sens commun et intelligence à la science est pour lui une illusion fatale.

"Il ne s'agit pas, comme dans la tradition épistémologique, de savoir lequel des deux est le "vrai" représentant de la "réalité". » (p. 189)

Dans la conception naturaliste de Dewey, science et sens commun sont deux modes d'accès au réel dont aucun n'a une réelle supériorité sur l'autre. Et la connaissance est elle-même un processus transactionnel naturel dans lequel le pratique et l'émotionnel ont une place. Cela ne signifie pas qu'il n'y ait pas de différence.

«Faire et connaître sont tous deux impliqués dans le sens commun et dans la science, impliqués si intimement qu'ils sont des conditions indispensables à leur existence. La différence entre sens commun et science n'est pas dans le fait que connaître serait important en science et pas dans le sens commun. La différence se situe dans la position de chacun par rapport à l'autre. Dans les soucis du sens commun, connaître est aussi nécessaire, aussi important que dans la science. Mais là, connaître est orienté par "l'attrait pour l'immédiat", le quoi et le comment de ce qui doit être étudié et appris, pour que les activités nécessaires de la vie quotidienne se poursuivent. La relation est inverse dans la science. On l'a déjà dit, faire et connaître sont également impliqués dans n'importe quelle technologie industrielle. Mais ce faire et ce connaître sont ici menés pour faire avancer le système de la connaissance et de la recherche. Dans chaque cas, faire est faire, et connaître est connaître. » (p. 188)
Ce qui fait donc la science est son action orientée et guidée par le souci prioritaire de connaître, et donc par le développement de méthodes qui permettent de garantir des conséquences. Il faut donc qu'elle s'abstraie de considérations immédiates et pratiques qui restent pourtant en arrière-fond de ses interrogations.

« En tout cas, il est aussi dangereux que stupide d'ignorer que la pureté de la recherche est quelque chose qui suppose un effort et qui doit être entretenu par une attention scrupuleuse : cela implique de reconnaître que l'activité scientifique est une action qui émerge des intérêts humains primaires et qui y retourne. Car si ce sont les propres intérêts et préoccupations de l'état des savoirs qui orientent le choix des objets de recherche, ils ne sont pas les seuls. Les problèmes ne se définissent pas d'eux-mêmes, et la direction que prend la recherche est conditionnée par des facteurs humains qui procèdent de l'intérêt et des préoccupations dominants et qui influencent le choix des questions qui doivent être étudiées. » (p. 189)

Dewey traite alors de l'autonomie de la science, mais il ne la pose pas comme un fait épistémologique. C'est plutôt pour lui un idéal moral, normatif qui requiert attention et soin pour préserver l'activité de recherche des influences institutionnelles et notamment des intérêts économiques particuliers.

La science se spécifie par la recherche de relations stables, lesquelles, si elles sont validées, permettent de prédire, de prévoir des conséquences. Mais pour ce faire, elle doit isoler certains processus, les abstraire du monde mouvant et changeant des flux. Ce faisant, elle se donne les moyens d'agir mais, en même temps, elle se sépare de la vie ordinaire pour construire des objets différents, créer du nouveau et donc transformer le monde.

Mais ce processus d'abstraction qui caractérise la recherche, et qui conduit à traiter certains phénomènes - par observation, par expérimentation - consiste aussi à ignorer, à laisser de côté. De même d'ailleurs, le sens commun, pour résoudre pratiquement les questions qui le concernent, sélectionne ce sur quoi il peut agir, ce qu'il peut mobiliser comme ressources, ce sur quoi il veut porter.

"Une science est "à propos de quelque chose", une science "de quelque chose", mais en fait elle laisse de côté ce quelque chose, comme on l'a répété avec cet exemple de l'eau où les usages et plaisir de l'eau comme eau sont radicalement différents des usages et plaisir qu'offre le laboratoire quand il constitue $\mathrm{H}_{2} \mathrm{O}$ comme objet d'investigation. L'effet libérateur de l'abstraction, typique de l'activité scientifique, apparaît dans la transformation des affaires inscrites dans les préoccupations communes du fait du mouvement de retour des méthodes et résultats des recherches dans les usages, joies et souffrances quotidiennes, retour qui s'accompagne de changements dans les jugements, les émotions, les préférences et les aversions des hommes ordinaires dans le quotidien. » (p. 188) 


\section{Le retour au sens commun}

Dewey inscrit donc comme une sorte de nécessité l'interrogation sur les conséquences, sur les effets des créations scientifiques dans le monde commun. Car ces produits de l'invention scientifique affectent bien évidemment l'état des choses.

« [...] Il y a abondance de preuves de ce que le retour des résultats (qui se produit de manière de plus en plus rapide et avec une extension croissante) est un mélange de choses bonnes et de choses condamnables. Le problème est alors de savoir comment ces résultats de la science peuvent être orientés pour soit en minimiser les conséquences dangereuses, soit en intensifier et étendre les conséquences positives et, si possible, d'élucider la manière dont cela peut se faire. » (p. 189)

Au passage, Dewey conteste qu'on puisse attribuer à la science une sorte de volonté de maîtrise et de possession de la nature : il assure que la maîtrise est une conséquence (au sens de ce qui s'ensuit) de la connaissance, et même qu'il n'y a de connaissance que pour pouvoir agir, parce que connaître permet de prédire ; mais, pour lui, il faut distinguer cette possibilité d'action que donne la connaissance, du sens de cette maîtrise, bref du type d'usage qui en est fait. Cela incite et donne du sens à une réflexion sur la science et ses usages qui soit ancrée dans le sens commun.

«[...] la manière la plus simple de se sortir des divisions, des isolements, des partages qui gênent la vie humaine serait de traiter avec sérieux des intérêts, des soucis, des affaires, etc., de ce sens commun, pour autant que tout cela soit des transactions (a) qui sont constituées de l'indissoluble union de facteurs humains et non humains, (b) dans lesquelles les traits et caractéristiques qualifiés d'intellectuels et d'émotionnels sont loin d'être indépendants et isolés de préoccupations pratiques, et enfin (c) que ces transactions sont des choses faites et des choses à faire, facta et facienda, tout ce qui relève et est enveloppé de la seule et essentielle affaire - l'état et le cours de la vie comme un ensemble de transactions. » (p. 189)

Si l'interdisciplinarité est par définition une forme urgente de réflexivité, John Dewey nous pressait il y a près de septante ans de l'ancrer dans les « affaires » de la cité et de la centrer sur les conséquences de la science. C'est dire que, si l'interdisciplinarité met en présence des disciplines et des théories, son espace n'est pas seulement celui de la science mais aussi celui du sens commun qui prend au sérieux les affaires humaines. 